\title{
Migración de retorno y capital humano Return migration and human capital
}

\section{Carlos Nieto*}

\begin{abstract}
Resumen
El presente artículo se interesa en la relación entre migración y desarrollo. La afirmación de que los migrantes acumulan valioso capital humano durante la migración es uno de los fundamentos del discurso dominante impulsado por las instituciones internacionales. Sobre la base de un trabajo cualitativo con migrantes peruanos que han retornado de Italia (entrevistas realizadas en Italia y Perú) se relativizan estas afirmaciones y se intenta demostrar que la realidad es mucho más compleja y contradictoria. Nuestros resultados sugieren que no existe una evolución uniforme de las diferentes dimensiones del capital humano sino más bien una evolución asimétrica y contradictoria. Hemos identificado dos grupos de migrantes retornados en función de la acumulación de capital humano durante la migración. En el caso de trabajadores migrantes que realizan trabajos de poca cualificación no existe acumulación de capital humano susceptible de ser puesto en valor luego del retorno y que por el contrario se aprecia una pérdida absoluta y relativa del capital humano inicial.
\end{abstract}

\section{Palabras clave}

Perú, Italia, migración, retorno, capital humano.

\begin{abstract}
This present article is interested in the relationship between migration and development. The argument that migrants accumulate valuable human capital during migration is one of the pillars of the dominant rhetoric propagated by international institutions. On the basis of a qualitative work with Peruvian migrants returning from Italy (interviews held both in Italy y Peru), such statements lose force. We aim to show that reality is far more complex and contradictory. Our results suggest that there is no harmonious evolution of the various dimensions of human capital, but rather an asymmetric and contradictory one. We identified two groups of returning migrants under the aspect of the accumulation of human capital during migration. In the case of migrant workers carrying out low-skilled activities, there is no accumulation of human capital to be appreciated after they return, much to the contrary: one states the absolute and relative loss of their initial human capital.
\end{abstract}

\section{Key words}

Peru, Italy, migration, return, human capital.

Forma sugerida de citar: NIETO, Carlos. 2012. "Migración de retorno y capital humano", en: Universitas, enero-julio de 2012. Quito: Editorial Abya-Yala, pp. 53-67.

Economista. Doctor en Ciencias Sociales y Políticas de la Universidad Católica de Lovaina. Investigador del Centro de Estudios para el Desarrollo de esa misma universidad. 


\section{Introducción}

El discurso dominante sobre la relación entre migración y desarrollo es producido y reproducido por las principales instituciones del sistema internacional y en gran medida por la academia occidental. Este discurso afirma que los migrantes, además de capital financiero, adquieren capacidades, habilidades, ideas y nuevos valores en el extranjero lo cual, luego del retorno, los convertiría en actores del desarrollo. ${ }^{1}$

El análisis del discurso dominante demuestra que este se sustenta en tres hipótesis subyacentes centrales. La primera está relacionada con el capital financiero. Se afirma que los migrantes retornados llevan consigo los ahorros fruto de su trabajo en el extranjero. Este capital es invertido en diferentes actividades empresariales, las cuales dinamizan y desarrollan los espacios locales contribuyendo a la modernización económica. Los capitales repatriados por los migrantes de retorno serían entonces verdaderas fuentes de capital destinados a financiar el desarrollo. La segunda hipótesis se refiere al capital humano: el discurso dominante asume que durante su estadía en el extranjero el migrante adquiere nuevas habilidades, capacidades, destrezas y valores, los cuales podrían facilitar la modernización y el crecimiento económico de los países de origen. La tercera hipótesis hace referencia al capital social adquirido por los migrantes. Este capital social transnacional puede servir para desarrollar actividades empresariales a nivel internacional. Entonces, los migrantes retornados poseedores de estos tres tipos de capital se convierten en los nuevos actores y dinamizadores del desarrollo en sus países de origen.

El presente artículo se interesa en contrastar la segunda hipótesis del discurso dominante. La acumulación de capital humano es evidente en el caso de estudiantes que migran para realizar una formación especializada en el extranjero. Luego del retorno, estos podrán contribuir al desarrollo de sus países de origen siempre y cuando la transferibilidad del capital humano sea posible. Lo mismo pasa con los migrantes altamente cualificados que parten a países industrializados integrándose en tanto que funcionarios o científicos y que luego retornan llevando con ellos el know how adquirido.

1 Global Commission on International Migration: GCIM 2005, Organisation de Coopération et de Développement Economiques : OCDE 2008, Organisation Internationale pour les Migrations: OIM 2008, Bureau International du Travail : BIT 2010. 
Sin embargo, ¿qué pasa con los trabajadores migrantes? ¿aquellos que migran para trabajar y que, muchas veces a pesar suyo, no pueden realizar ningún tipo de formación profesional?

Desde la academia occidental se asume también que los migrantes adquieren nuevas capacidades, habilidades, ideas y valores en el extranjero (ThomasHope 1999, Ghosh 2000, McCormick y Wahba 2001). Sin embargo, la mayor parte de los trabajos empíricos sobre la migración de retorno no permiten conocer de manera precisa, en el caso de trabajadores migrantes, cómo se realiza el proceso de adquisición de capital humano durante la migración. Se han avanzado en algunas nociones como la 'exposición' al funcionamiento de empresas en nuevos contextos económicos (BIT 2010). Otro argumento indica la existencia de externalidades en la función de aprendizaje (OCDE 2008). Los migrantes podrían igualmente favorecer la 'adopción' y la 'transferencia' de nuevas tecnologías a sus países de origen (Gmelch 1980).

Algunos estudios muestran los límites de esta visión dominante. Estos argumentan que los migrantes adquieren muy poco capital humano, debido a que realizan trabajos no cualificados, que no les permite obtener mayores conocimientos. Se afirma además que incluso en el caso en que los migrantes han adquirido nuevos conocimientos en el extranjero, es poco probable que estos puedas ser utilizados de manera productiva en sus países de origen (Enchautegui 1993, Muschkin 1993, Co y otros 1998).

Es pertinente preguntarse entonces sobre los procesos de acumulación de capital humano durante la migración. En este artículo nos interesa conocer: si efectivamente existe acumulación de capital humano durante la migración ¿cuál es el comportamiento de las tres dimensiones del capital humano? ¿se acumulan por igual? ¿por qué algunos migrantes adquieren mayor capital humano que otros? ¿de qué depende?

\section{Construcción de datos}

Este artículo estudia la migración de retorno de quillabambinos provenientes de la ciudad de Turín en Italia. La ciudad de Quillabamba esta ubicada al norte del departamento del Cusco en el sur del Perú. La ciudad cuenta con una población de 25.584 habitantes. A partir de fines de los años ochenta la población de esta ciudad conoce un importante proceso de migración hacia el norte 
de Italia. A partir de los años 2000, algunos migrantes comienzan a retornar y se reinstalan en la ciudad de Quillabamba y Cuzco.

El presente artículo utiliza la información cualitativa recolectada a través de 43 entrevistas a migrantes quillabambinos. Se han efectuado 18 entrevistas en Quillabamba con migrantes retornados y 25 entrevistas en Turín con migrantes que tienen deseo de retornar pero que no tienen fecha de retorno definida. Las entrevistas se han realizado entre julio 2008 y mayo 2010.

Nuestros resultados nos permiten delinear algunas características generales de los migrantes entrevistados. La primera característica es la posesión de capital humano medio y elevado, acumulado antes de la migración. Algunos poseen titulo universitario, otros tienen estudios universitarios no concluidos y otros estudios superiores no universitarios concluidos y no concluidos. La segunda característica es que el trabajo realizado en Italia es inferior a sus cualificaciones profesionales. Los entrevistados trabajan o han trabajado como empleadas domesticas, cuidado de ancianos, obreros, choferes, soldadores.

\section{La migración peruana en Italia y el retorno}

La migración de trabajadores peruanos a Italia comenzó a finales de los años ochenta. La mayoría de estos migrantes se ha ubicado en el norte de Italia, fundamentalmente en las ciudades de Milano, Turín y Génova. Considerando que la migración intercontinental demanda una elevada movilización de capital social y un alto costo financiero, no son los más pobres los que migran a Europa: la migración a Italia es una estrategia familiar principalmente de las clases medias urbanas empobrecidas.

El nivel de educación de los migrantes en Italia es medio y alto. Como referencia podemos tomar las estadísticas oficiales, ${ }^{2}$ donde se aprecia que el 10,4\% de los migrantes peruanos son profesionales universitarios, $28,2 \%$ son estudiantes, $13,3 \%$ son empleados de oficina y $5,7 \%$ son técnicos y profesionales de nivel medio, haciendo un total de 57,6\% con estudios técnicos y universitarios.

Según el Consulado del Perú en Milán (2008) “el grado de instrucción de los peruanos migrantes no guarda relación con el trabajo que efectúan en Ita-

2 Instituto Nacional de Estadística e Informática: INEI - OIM - Dirección General de Migración y Naturalización: DIGEMIN 2008 
lia”. Los migrantes peruanos en Italia se insertan principalmente en el sector de servicios poco cualificados. El Consulado del Perú en Milán $(2008,10)$ ha clasificado por orden de importancia los trabajos que desempeñan los migrantes en esa ciudad: empleados de limpieza, empleadas domésticas o empleados en general; operarios, almaceneros, albañiles; cuidado de ancianos y niños; choferes; enfermería; pintor, soldador; gastronomía, cocinero y ayudante de cocina; comerciantes, vendedores; estilistas, cosmetólogos; electricidad; mecánicos, entre otros. De acuerdo con estos datos el $81 \%$ de los migrantes están ocupados como empleados domésticos y de limpieza, cuidado de ancianos y niños y operarios albañiles.

Los datos estadísticos de 2007 sobre la migración de retorno desde Italia (INEI - OIM 2009) indican que el 32,2\% de retornados cuenta con estudios superiores universitarios completos, el 9,0\% con estudios superiores universitarios incompletos, el $11,5 \%$ con estudios superiores no universitarios completos, y el $15,4 \%$ con estudios superiores no universitarios completos. Lo que significa que siete de cada diez migrantes retornados de Italia cuenta con estudios superiores.

Los migrantes quillabambinos en Turín no escapan a esta realidad. La mayoría de ellos pertenecen a estratos medios de su contexto de origen, cuentan con estudios superiores universitarios y no universitarios y en Italia están integrados en el sector de servicios poco cualificados (Nieto 2012).

\section{Breve conceptualización operativa de capital humano}

El concepto de capital humano es usado en economía desde los años 60 (Becker 1964). Con el paso del tiempo, la teoría del capital humano se ha modificado y complejizado. Actualmente no existe una definición única de capital humano. Para el Banco Mundial, el capital humano es "el conjunto de conocimientos, competencias y experiencias que poseen los individuos y que los convierte económicamente productivos". ${ }^{3}$

Sobre la base de esta definición propongo operacionalizar este concepto estableciendo tres componentes o dimensiones. Utilizaremos este concepto

3 http://www.worldbank.org/depweb/beyond/beyondfr/glossary.html 
operativo para analizar la adquisición de capital humano en el caso de los migrantes peruanos retornados de Italia.

- Los saberes y conocimientos: Los saberes son definidos como un conjunto de conocimientos reproductibles, adquiridos por medio de los estudios, representados por diplomas, títulos académicos y certificados. A esta dimensión del capital humano la llamaremos capital humano formal (Chf).

- Las competencias, entrenamientos, habilidades y capacidades: Son un conjunto de conocimientos y capacidades adquiridos generalmente a través de la práctica y aplicables al ejercicio de una actividad productiva. A esta dimensión la llamaremos capital humano competencias (Chc).

- Las experiencias: Se refieren a las experiencias de trabajo y de vida de cada persona pudiendo aportar a un individuo en su productividad. Capital humano experiencias (Chx).

\section{Presentación de resultados}

Podemos afirmar estar frente a una situación compleja y tal vez contradictoria. Analizando las tres dimensiones del capital humano, vemos que no existe una evolución uniforme sino más asimétrica de cada una de estas. Hemos identificado dos grupos de migrantes retornados en función de la acumulación de capital humano durante la migración.

\subsection{Primer grupo: pérdida de capital humano formal, no acumula- ción de competencias y acumulación de experiencias}

Este grupo no ha acumulado capital humano formal (Chf). Hemos identificado cuatro elementos explicativos que están al origen de la dificultad, léase, imposibilidad de acumular capital humano en Italia: barreras administrativas en el reconocimiento de grados y títulos, el tipo de trabajo y las precarias condiciones laborales, la deficiente inserción social del migrante y la falta de interés de algunos migrantes en realizar estudios complementarios.

Las barreras administrativas a la convalidación de grados y títulos limitan fuertemente el deseo de ciertos migrantes de efectuar algún curso de capacita- 
ción. ${ }^{4}$ Los migrantes entrevistados han calculado que el trámite administrativo, tanto en Perú como en Italia, puede tomar hasta un año y el costo del mismo podría ascender a tres mil dólares americanos. El trámite de convalidación de grados y títulos conlleva un esfuerzo importante en tiempo y en dinero y es justamente lo que los migrantes menos tienen. Debido a esta dificultad los migrantes que tienen el deseo de realizar estudios en Italia no logran este objetivo.

El tipo de trabajo efectuado influye en la no acumulación de capital humano formal. La mayoría de migrantes efectúan trabajos no cualificados y muy por debajo de sus capacidades. Los migrantes ocupan empleos con alto grado de precariedad, socialmente aislantes y con malas condiciones laborales. Por ejemplo, en el caso de los migrantes que trabajan cuidando ancianos, la precariedad se manifiesta en la duración del empleo, el migrante no tiene ninguna certeza del tiempo que podrá permanecer en ese trabajo, el tiempo de permanencia depende de la salud de la persona bajo su responsabilidad. Un trabajo puede durar unos años o solo unos meses. Esta precariedad y falta de seguridad laboral dificulta la realización de proyectos a mediano plazo. Otros entrevistados manifiestan tener horarios nocturnos, por lo general este tipo de empleo se da en fabricas. El hecho de trabajar durante la noche y descansar en el día tiene consecuencias a nivel del aislamiento social del migrante.

Las características mencionadas, sumadas al prejuicio de la sociedad receptora sobre los migrantes, van a limitar la inserción social del migrante. La mayoría de migrantes andinos son integrados a los niveles más bajos de la estructura social italiana. Esta situación podría cambiar con el paso del tiempo, sobre todo con la denominada segunda generación. Experiencias de migraciones más antiguas así lo evidencian.

El cuarto elemento a mencionar es la falta de deseo por realizar estudios durante la migración, en efecto, no todos los migrantes tienen interés en mejorar su capital humano formal, es más, podemos decir que la mayoría de los trabajadores migrantes no tiene el proyecto de realizar estudios complementarios. Los trabajadores migrantes que tienen como objetivo trabajar unos años en el extranjero, acumular ahorros y retornar a su país de origen no tienen in-

$4 \quad$ Este análisis no incluye la convalidación y reconocimiento del título profesional de Enfermera. Esta profesión goza de preferencias administrativas para su convalidación en Italia. Existe un número considerable de enfermeras trabajando en Italia con sus títulos profesionales debidamente convalidados y reconocidos. 
terés en efectuar estudios de capacitación. Este interés aparece más bien en los migrantes que desean quedarse a vivir en Italia y buscan una movilidad social ascendente. También se observa este interés en los llamados migrantes de segunda generación.

La confluencia de estas características configura una situación tal que hace muy difícil acumular capital humano formal durante la migración.

Además de la no acumulación de capital humano formal que hemos explicado, nuestras entrevistas nos permiten afirmar que en la mayoría de los casos se da una pérdida absoluta y relativa de capital humano formal durante la migración. La pérdida absoluta se refiere al olvido y a la falta de puesta en práctica de los conocimientos profesionales adquiridos antes de la migración. Hemos entrevistado profesionales universitarios que trabajan como choferes, otros como estibadores de mudanza, cuidado de ancianos, empleadas domesticas, etc. Estos profesionales manifiestan que después de varios años de falta de práctica en sus respectivas profesiones han olvidado muchos de sus conocimientos. A este fenómeno lo llamamos pérdida absoluta de capital humano como consecuencia de la migración.

La pérdida relativa de capital humano significa la pérdida de ventajas competitivas en relación a los otros profesionales que no han migrado. Por ejemplo, podemos mencionar el caso de un ingeniero químico que ha trabajado como cargador y chofer en una cooperativa dedicada a la mudanza. Luego de su retorno al Perú, nuestro entrevistado constató que durante los diez años que estuvo trabajando en Italia acumulando capital financiero, sus compañeros de universidad y colegas ingenieros han logrado realizar estudios de posgrado, maestrías, estudios de segunda especialización, etc. y han ganado experiencia profesional. Nuestro entrevistado tiene el deseo de reintegrarse al mercado laboral en el Perú en tanto que ingeniero químico, pero ha advertido que tiene una desventaja competitiva en razón de la pérdida relativa de su capital humano durante la migración.

En relación a la segunda dimensión del capital humano, observamos que este primer grupo no ha logrado adquirir competencias, entrenamientos, habilidades y capacidades susceptibles de ser capitalizados luego del retorno al Perú. La explicación de la no-acumulación de capital humano por competencias (Chc) está en el tipo de trabajo realizado por los migrantes en Turín. La mayor parte de los migrantes peruanos en Italia se dedican al cuidado de ancianos, al trabajo doméstico y a otros trabajos manuales de poca cualificación. 
La integración de los migrantes a estos segmentos del mercado laboral limita la adquisición de competencias, entrenamientos y habilidades capaces de ser puestos en valor después del retorno al país de origen.

Veamos el ejemplo de una profesora de educación inicial, nuestra entrevistada tiene un contrato de duración indefinida con el Ministerio de Educación del Perú, durante la migración pidió licencia indefinida sin goce de haber. Durante los 9 años que permaneció en Italia ha trabajado como empleada de limpieza, luego del retorno la entrevistada ha retomado su puesto de profesora. Las capacidades y competencias que la entrevistada hubiera podido adquirir durante su trabajo en Italia no son valorizables en el desempeño de su actividad profesional después del retorno.

La evaluación de la tercera dimensión del capital humano de este primer grupo muestra una evolución positiva (Chx). Todos los entrevistados concuerdan en afirmar que la experiencia de vida en Italia es positiva. El conocimiento de otra cultura, el haber adquirido otro idioma, el haber vivido y trabajado en otro contexto sociocultural son valorados como positivos. En algunos casos, el hecho de dejar la familia, la experiencia de vivir solos y 'salir adelante' son también valorados. Según los migrantes, la experiencia migratoria, a pesar que pueda ser en algunos casos difícil y dolorosa, es definitivamente una experiencia enriquecedora. Queda por analizar y demostrar ¿cómo esta experiencia de vida, valorada como positiva, influye en la productividad de los migrantes retornados? Sería interesante comparar la productividad de los migrantes retornados con la de los no migrantes y analizar en qué medida la migración modifica la productividad.

La evolución asimétrica y contradictoria de las diferentes dimensiones del capital humano de este primer grupo puede ser representada en el siguiente grafico. 


\section{Grafico 1}

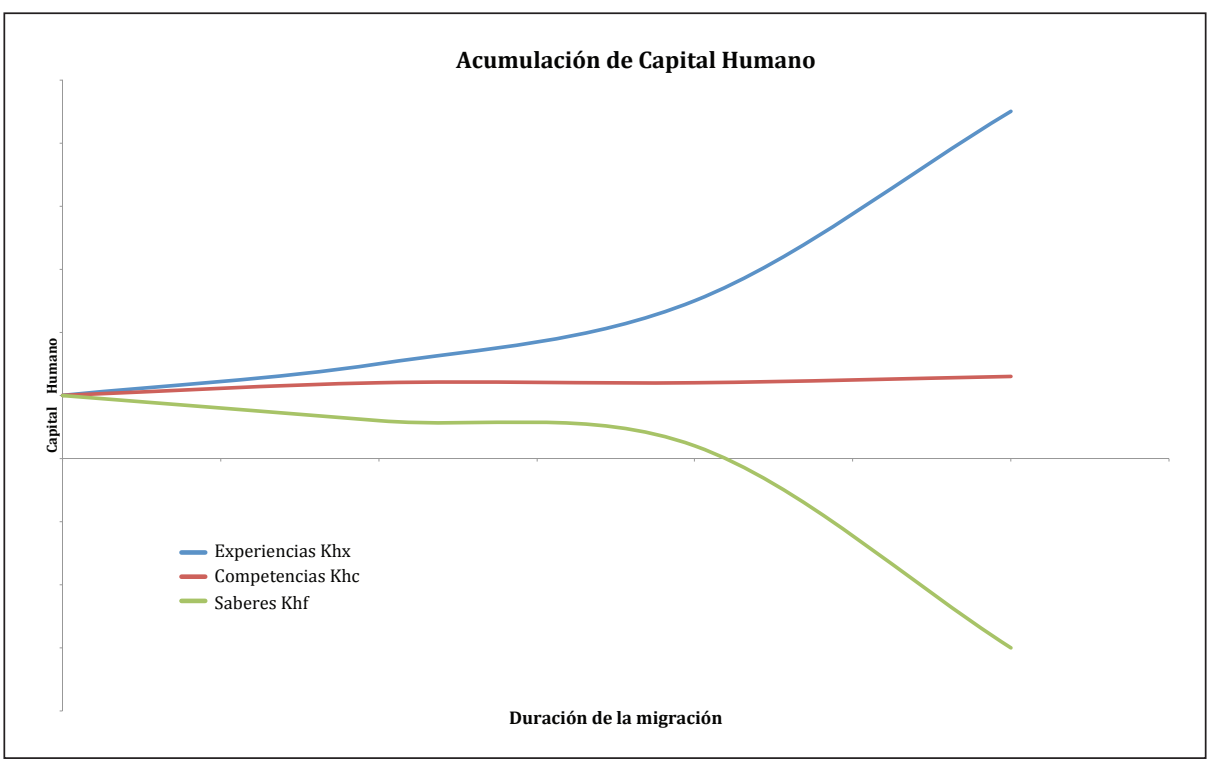

Elaboración propia

\subsection{Segundo grupo: pérdida de capital humano formal, acumula- ción de competencias y acumulación de experiencias.}

Este segundo grupo presenta las mismas características que el primero en cuanto al comportamiento de la dimensión formal (Chf) y la dimensión experiencias (Chx) del capital humano. Sin embargo, los migrantes de este grupo manifiestan un incremento en la segunda dimensión del capital humano: competencias, entrenamientos, habilidades y capacidades (Chc). La ganancia de capital humano, en la dimensión competencias, es obtenido por una combinación de tres elementos interdependientes: el tipo de trabajo efectuado en Italia, las condiciones de inserción laboral y la consecuente integración social en el país de acogida. En este grupo encontramos migrantes que han efectuado otro tipo de trabajos que aquellos habitualmente reservados a los migrantes peruanos (empleada domestica, cuidado de adultos mayores, etc.). Las condiciones de 
inserción laboral son también menos precarias que las del primer grupo y la integración social mantiene una correlación con las buenas condiciones laborales y la calidad del puesto de trabajo.

A título de ejemplo podemos mencionar el caso de un migrante retornado, que gracias sus contactos familiares logra obtener un puesto de supervisor en una pequeña planta de tratamiento industrial de telas. Nuestro entrevistado es ingeniero agrónomo de profesión, una parte de su familia vive en Italia. La hermana del entrevistado trabaja para una familia que posee una pequeña empresa de tratamiento industrial de telas. Gracias a la buena relación de la hermana con la familia propietaria, nuestro entrevistado logra un contrato de trabajo y viaja a Italia para integrarse a la empresa. Debido a su empeño y dedicación en el trabajo, a su demostrada capacidad y al contacto directo con la familia propietaria, logra poner en valor sus estudios universitarios previos y asciende al puesto de supervisor de procesos. Con el paso del tiempo el entrevistado se convierte en 'el brazo derecho' del dueño de la empresa. Esta adecuada integración laboral le permite un salario por encima del promedio del resto de migrantes, así como una integración social juzgada como aceptable. Luego de varios años de trabajo en la empresa, el entrevistado pierde el interés, la motivación y el entusiasmo por su trabajo, en esas circunstancias decide volver al Perú e instalarse en Quillabamba. En el momento de la entrevista el migrante retornado poseía dos pequeñas empresas en esa ciudad.

Cabe preguntarse, sabiendo que no existe una industria similar en esta ciudad, ¿en qué medida las competencias adquiridas en tanto que supervisor de una planta de tratamiento industrial de telas pueden ser utilizadas de manera productiva en Quillabamba? La respuesta puede ser obvia. Sin embargo, durante las entrevistas a este grupo de migrantes dos elementos emergieron de las conversaciones: la autoestima y la seguridad en sí mismos. En efecto, más allá de las habilidades y capacidades técnicas adquiridas por estos migrantes, se evidencia una elevada autoestima y una alta seguridad personal. A pesar de que estos elementos puedan ser de alguna forma subjetivos y difíciles de medir, creemos que la adquisición o el fortalecimiento de estos dos elementos son un aporte importante de la migración a este grupo de migrantes.

El grafico siguiente muestra el comportamiento del capital humano de este segundo grupo de migrantes. 
Gráfico 2

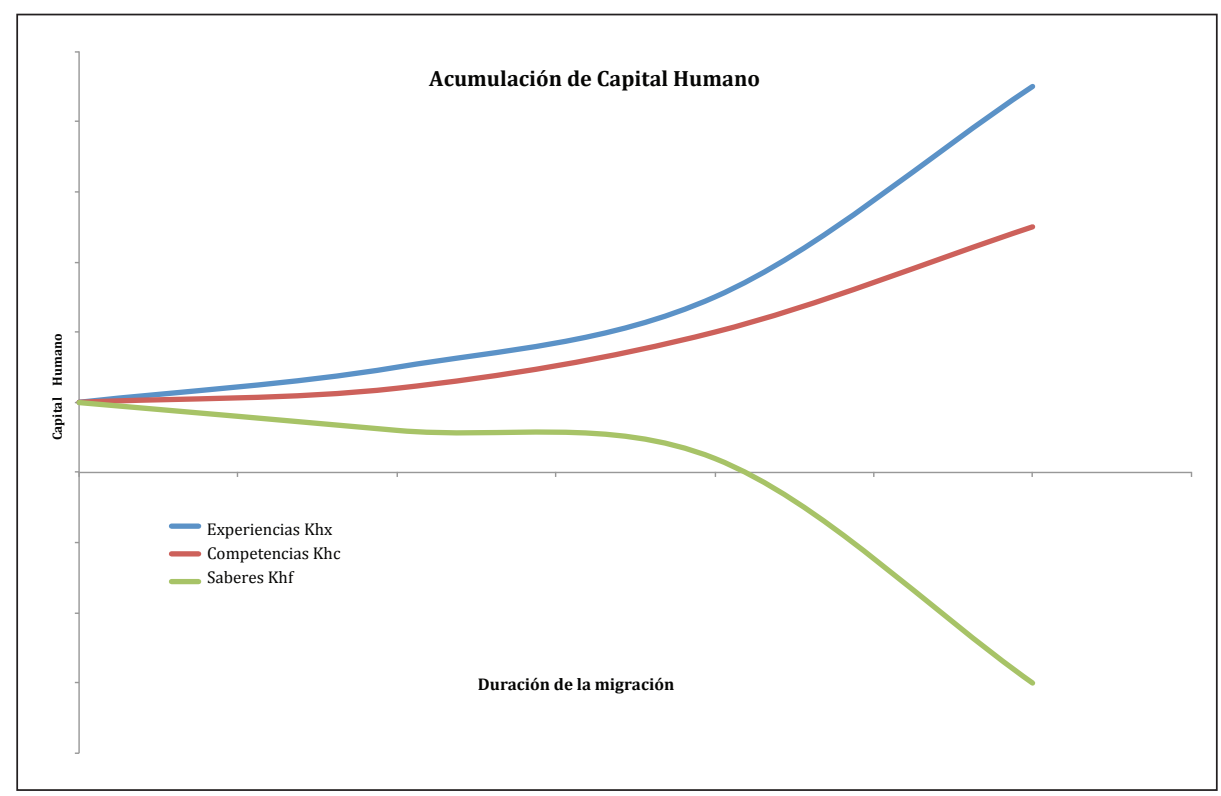

Elaboración propia

La mayoría de los migrantes retornados entrevistados están representados en el primer grupo, solo una pequeña minoría hacen parte del segundo grupo.

\section{Discusión}

Podemos afirmar que estamos ante una situación compleja y divergente. Analizando las tres dimensiones del capital humano, observamos que no existe una evolución uniforme sino más bien que esta es asimétrica y contradictoria.

Los resultados de nuestro trabajo contradicen la hipótesis subyacente del discurso dominante sobre la adquisición de capital humano durante la migración. Según esta hipótesis, los migrantes adquieren nuevos conocimientos, competencias, habilidades, capacidades, destrezas y valores durante la migración. Nuestros resultados sugieren que, en el caso de trabajadores migrantes que se integran o son integrados en trabajos poco cualificados, no existe acumulación de capital humano susceptible de ser puesto en valor luego del retorno de 
los migrantes y que, por el contrario, se aprecia una pérdida absoluta y relativa del capital humano inicial.

Sin perjuicio de lo último mencionado, existe un pequeño grupo de migrantes que efectivamente ha logrado una acumulación parcial de capital humano. Las competencias acumuladas durante la migración podrían servir, en determinadas circunstancias, para mejorar la capacidad competitiva de los migrantes retornados.

En todos los casos, la dimensión de las experiencias muestra una evolución ascendente. La experiencia migratoria es valorada como positiva por todos los migrantes entrevistados. Esta sería la dimensión que se enriquece gracias a la 'exposición' a nuevos contextos económicos. Sin embargo, no sabemos en qué medida esta experiencia de vida permite al migrante retornado incrementar sus capacidades competitivas. Dicho de otro modo, no sabemos si la experiencia migratoria puede ser una externalidad positiva en la productividad. Es necesario profundizar el tema con un estudio comparativo que mida la productividad de los migrantes retornado y los no migrantes.

Nuestros resultados delinean una realidad mucho más compleja y contradictoria que la presentada por el discurso dominante. Nuestro trabajo demuestra la necesidad de relativizar la hipótesis de la acumulación de capital humano durante la migración del discurso dominante y resalta la necesidad de profundizar el análisis de las diferentes dimensiones del capital humano tomando en cuenta la diversidad y singularidad de las trayectorias migratorias.

El análisis comparativo de los dos grupos presentados nos permite subrayar dos elementos que pueden condicionar e influenciar la adquisición de capital humano durante la migración.

El primer elemento es la posesión y la buena gestión de redes y capital social. Hemos observado que en algunos casos las redes y los contactos han sido fundamentales para que el migrante se inserte en otros espacios laborales. Sin embargo, y de manera contradictoria, las redes también pueden servir para perpetuar a los migrantes en determinados espacios laborales. Esta aparente contradicción resulta sumamente interesante puesto que nos da pistas para pensar que la migración en sí no sería un medio para incrementar el capital humano, sino que este incremento estaría ligado a la posesión de capital humano y social previo a la migración y a la selectividad de la migración. Los migrantes que inicialmente poseen un pobre capital social, una vez en su destino se integrarían en segmentos laborales relegados a migrantes. En cambio, los migrantes 
que inicialmente poseen un elevado capital social y óptimas redes migratorias, una vez en su destino, logran poner en valor su capital humano inicial y se integrarían a segmentos laborales más expectantes y con mayor capacidad movilizadora.

El segundo elemento tiene que ver con las oportunidades de integración laboral y social que ofrece la sociedad o el grupo receptor. En la medida en que la sociedad o grupo receptor otorgue mayores posibilidades de inserción laboral y social, reconozcan el capital humano inicial de los migrantes, aparentemente, mayores son las posibilidades de adquisición de nuevo capital humado durante el tiempo que dure la migración. Inversamente, los países que limitan la integración y el desenvolvimiento socio profesional de los migrantes, subutilizando el capital humano inicial de estos, aparentemente, limitan también la adquisición de nuevo capital humano.

\section{Bibliografía}

Banco Mundial, Glossaire $<\underline{\text { http://www.worldbank.org/depweb/beyond/beyondfr/glossary.html }>\quad[28}$ de febrero de 2012].

BECKER, Gary

1964 Humain Capital : A Theoretical and Empirical Analysis, with Special Reference to Education, University of Chicago Press.

BIT,

2010 Faire des migrations un facteur de développement. Une étude sur l'Afrique du Nord et l'Afrique du l'Oeste, Institut international d'études sociales, Genève.

CO, Catherine, M. Yun e I. Gang.

1998 "Returns to Returning: Who Went Abroadand What Does It Matter?", in Discussion Paper, No. 19, August 1998, IZA.

Consulado del Perú en Milán

2008 "Perfil de la migración peruana en las regiones italianas de Lombardía y Emilia Romagna, 2006-2008”, Milán.

ENCHAUTEGUI, María

1993 "The Value of U.S. Labor Market Experience in the Home Country: The Case of Puerto Rican Return Migrants", in Economic Development and Cultural Change, Vol. 42, N 1 (Oct., 193), pp. 169-191. 


\section{GCIM}

2005 Les migrations dans un monde interconnecté: Nouvelles perspectives d'Action. Rapport de la Commission Mondiale sur les Migrations Internationales, Genève.

GHOSH, Bimal

2000 Return migration. Journey of hope or despair?, Geneva, OIM.

GMELCH, George

1980 "Return Migration”, in Annual Review of Anthropology, Vol. 9, pp. 135-159.

INEI - OIM - DIGEMIN

2008 Perú; Estadísticas de la migración internacional de peruanos, 1990-2007, Lima, OIM.

\section{INEI - OIM}

2009 Migración internacional en las familias peruanas y perfil del peruano retornante, Lima.

MCCORMICK, B. y J. Wahba

2001 “Overseas Work Experience, Savings and Entrepeneurship Amongst Return Migrants to LDCs", University of Southampton, UK.

Muschkin, Clara

1993 "Consequences of Return Migrant Status for Employment in Puerto Rico", in Nieto, Carlos International Migration Review, Vol. 27, No. 1 (Spring, 1993), pp. 79-102.

2012 Relation entre migration de retour et développement, Analyse du capital économique, humain et social chez les migrants retournés, entre le discours et la pratique, Louvain-la-Neuve, Presses Universitaires de Louvain. (En prensa)

OCDE

2008 Perspectives des migrations internationales. Rapport annuel, Edition SOPEMI.

OIM

2008 "Dialogue international sur la migration 2008. Migration de retour: Defies et opportunites. Principes politiques clés de la migration de retour", Genève.

Thomas-Hope, Elizabeth

1999 "Return Migration to Jamaica and its Development Potential”, in International Migration, Vol. 37 (1), p. 183-207.

Envío 13 de febrero de 2012 - Fecha de aceptación 7 de marzo de 2012. 